\title{
Bioética de la atención de enfermería al enfermo terminal
}

\author{
Francisco Javier León Correa* \\ Doctor en Filosofía. Director Grupo de Investigación en Bioética de Galicia, España. Profesor del Centro de Bioética de la \\ Universidad Católica de Chile. Santiago de Chile. \\ Recibido: 20-05-03; Aceptado: 15-07-03
}

\section{Introducción}

Aunque parte importante de las decisiones que han de tomar los equipos de salud en la atención a enfermos terminales corresponde al médico o al equipo médico, no es menos cierto que las consecuencias de esas decisiones afectan de manera muy clara el quehacer diario y la conciencia de las enfermeras que deben cuidar y atender a esos pacientes. Y, por otra parte, los cuidados de enfermería que deben darse a los enfermos terminales también plantean sus propias tomas de decisiones éticas que han de saber resolver -o intentar resolver- las propias enfermeras. Además, en la fase final de la vida "disminuye lentamente la función del médico y se hace más importante la de la enfermera: pasa a ser una importante fuente de información y consulta, y toma cada vez más la iniciativa» (1).

Como señalan expertos en cuidados paliativos, «el médico, cuando considere que el proceso es irreversible y terminal según los indicadores científicos objetivos, abandonará la idea de curar y se empleará a fondo en el oficio "muy exigente de ciencia, competencia y humanidad, que consiste en aliviar y consolar, en procurar su mayor bienestar material y espiritual, así como prestar su apoyo a los allegados de éste" (1). Desde el punto de vista clínico, es bien conocido que los pacientes que son ayudados a enfrentarse con la muerte y a sobrellevar ese conocimiento, atraviesan esta etapa con más paz y con menos síntomas de alteraciones del comportamiento» (2).
La mayoría de estos pacientes no fallece de forma súbita, sino que normalmente entra en un período de deterioro gradual muy importante con disminución progresiva del nivel de conciencia, cambios bruscos o crisis con fallos orgánicos multisistémicos, etc. El tiempo que transcurre desde el inicio del coma a la muerte es muy variable y va desde unas pocas horas a un par de semanas, pero generalmente ocurre en 24-48 horas. Este sería el período propiamente terminal del enfermo, aunque aquí utilizaremos un concepto más amplio que puede comenzar cuando se diagnostica una enfermedad o situación crónica irreversible, que va a causar la muerte del paciente. Son situaciones que producen un gran impacto emocional tanto en el paciente como en la familia y en el propio equipo sanitario que ha estado intentando su curación, el cual debe cambiar desde ese momento el enfoque y la finalidad de su actuación (3).

\section{Preparar al paciente para enfrentar la muerte con dignidad}

Es éste uno de los cometidos de todo el equipo sanitario, que debe realizarse de acuerdo con la familia y con las sucesivas etapas emocionales de adaptación que se presentan en mayor o menor medida en todos los casos: deseos de conocer o de confirmación del diagnóstico, shockcon fuerte conmoción interna, fase de negación, enfado, negociación o pacto, depresión, resignación y aceptación final (4).

\footnotetext{
* Correspondencia F. León: E-mail: gibioetica@edunet.es / www.unicolmayor.edu.co
} 
El paciente terminal experimenta una serie de inquietudes con preguntas que en ocasiones no se atreven a hacer ni a sí mismos; temores que expresarán en la medida en que encuentren profesionales de la salud a su lado que sean sensibles y comprendan lo que subyace en sus comentarios y dudas; dependencias físicas y psíquicas que pueden desmoralizarle y hacerle perder su propia autoestima y dignidad personal; búsqueda de un sentido: encontrar en medio de la desesperanza y depresión un sentido de lo que les ocurre, el sentido del sufrimiento, del dolor y, en definitiva, de su propia muerte. Pueden y deben encontrar en los profesionales sanitarios que les atienden una gran ayuda para adaptarse a ese proceso del morir, a esa despedida de sus seres queridos, antes de entrar en la inconsciencia; y los médicos y enfermeras deben estar preparados, profesionalmente también, para prestar este cuidado sanitario, psíquico y plenamente humano, en condiciones más difíciles de atención al paciente (5).

En definitiva, se trata de saber disponer al paciente para una muerte afrontada con dignidad. Para esto es necesario resolver una serie de puntos (6):

1. Lo primero es saber que se está muriendo. De este modo, procurará resolver sus asuntos, tomar sus disposiciones, cumplir sus promesas o simplemente despedirse.

2. Es necesario que el enfermo pueda seguir siendo, en cierto modo, agente, y no simplemente paciente. Por ello debe intervenir en las decisiones que se deban tomar sobre su enfermedad o sobre su persona. El paciente debe recibir verdadera información acerca de las decisiones y procedimientos que le afecten a él y a su bienestar y debe consentirlos antes de que se lleven a cabo.

3. Hay que preservar en la medida de lo posible la autonomía y autocontrol del paciente. La sensación de menor dignidad resulta de la excesiva dependencia y la pérdida de control de las funciones orgánicas.

Universidad Colegio Mayor de Cundinamarca - DIUM
4. Deberá mantener en la medida de lo posible ciertas actividades y relaciones familiares, sociales y profesionales. Sentirse persona implica ser capaz de relacionarse. Si el enfermo nota que es dejado de lado, que se evitan sus preguntas, que no es tocado al saludarle, etc., notará que pierde dignidad, no se sentirá tratado como persona. Una de las ventajas del sistema Hospiceanglosajón es que facilita precisamente una mayor y libre interacción del enfermo con familiares, amigos, médicos, etc.

5. Se debe proteger la pérdida de la imagen corporal. Hay que evitar que el enfermo se sienta desfigurado o depauperado.

6. El sentirse persona es a menudo mantenido por consideraciones transcendentes. Al enfermo se le debe facilitar el apoyo espiritual si lo desea.

\section{2. Ética del tratamiento paliativo del dolor}

Cuando ya no es posible curar al paciente, queda en primer plano el aliviar su sufrimiento, que no sólo es éticamente obligatorio sino que, además, justifica plenamente las actuaciones paliativas, que «son válidas, satisfactorias y gratificantes si consiguen tal finalidad» (7).

El profesional sanitario que trata enfermos en situación terminal tiene obligación de estar bien entrenado en el manejo de los diversos niveles de analgesia y aplicar en cada caso el conveniente para la situación del enfermo. En caso de que no consiga un eficaz control del dolor o se requieran técnicas especializadas (neurocirugía, radioterapia, neuroanestesia, etc.), debe poner al enfermo en manos del especialista indicado en su caso.

Cuando el enfermo se encuentra moribundo y el objetivo sea conseguir el máximo control del dolor, se pueden utilizar pautas de sedación en las que exista la capacidad de despertar y llevar o seguir una conversación lúcida.

En términos generales, existe la obligación ética de no privar de la conciencia de sí mismo sin verdadera 
necesidad. Sin embargo, cuando se pretende la supresión de la sensación dolorosa, si existen motivos serios y las circunstancias no imponen lo contrario, se puede evitar el dolor, aunque la narcosis lleve consigo una supresión o disminución de la conciencia.

Si de la aplicación de algún fármaco se siguiera un acortamiento de la vida como efecto no buscado, tampoco habría inconveniente en administrarlo. De todos modos, en la bibliografía especializada actual, no se cita ese efecto entre los adversos que se producen durante el uso prolongado de opiáceos. Sin embargo, algunos autores anotan que, por su efecto depresor del centro respiratorio, podrían facilitar la aparición de infecciones respiratorias. Por el contrario, otros señalan que globalmente los opiáceos alargan el tiempo de vida al paciente: «evidencias circunstanciales, sugieren que el uso correcto de la morfina prolonga la vida del paciente con cáncer en la medida en que se ve libre del dolor, en mejor situación para descansar, dormir y comer y, generalmente, se siente y está más activo» (8).

En resumen, lo que se pretende desde los cuidados paliativos «es dar la atención adecuada al enfermo, que le lleve a tener la mejor calidad de vida posible y que le permita llegar al desenlace final de su proceso en una situación de comprensión y ayuda, respondiendo de forma profesional a las necesidades físicas, emocionales, sociales y espirituales, dando prioridad a los objetivos del propio enfermo, tanto por parte de los profesionales como de su familia, a la que se le ofrecen los medios y ayuda necesarios para llevarlo a cabo. En un ámbito hospitalario o mejor aún, en su propio domicilio» (9).

\section{Cuidados mínimos y medidas de} soporte vital para pacientes en cuidados intensivos

El enfermo en situación terminal, ingresado en una UCI, sigue precisando de cuidados médicos y atención de enfermería (10). Las manifestaciones clíni- cas que requieren tratamientos y cuidados específicos durante la enfermedad terminal son muy variadas. Ciertamente, la administración de analgésicos, sedantes, antibióticos de uso común, transfusiones y oxigenoterapia, así como la colocación de sondas, drenajes y venopunción, son de uso corriente. Los cuidados otorgados a un paciente en estado terminal no son aplicados como medios para preservar la vida, sino que se aplican en razón de una asistencia éticamente obligada.

Tal y como se expresó en la «Declaración de Ética en Medicina Crítica y Cuidados Intensivos», «los intensivistas debemos ser conscientes de que este desiderátum (la reincorporación del paciente al seno de sus familias y de la sociedad) no es lograble en todos los pacientes. En algunos casos la recuperación es sólo parcial, y en otros será la muerte el resultado. Tener clara esta realidad es muy importante a fin de evitar el exceso de terapéutica o la prolongación innecesaria del morir» (11). También es muy interesante el análisis de las peculiaridades en cuidados intensivos pediátricos, y la necesidad de definir bien la situación crítica -tanto en adultos como en niños- que exige la permanencia en la UCI, de la situación grave crónica, que puede y éticamente sería más conveniente que fuese atendida en hospitalización domiciliaria (12).

Con independencia de su salud o condición, toda persona tiene derecho a recibir unos cuidados mínimos, si no se quiere menospreciar su dignidad. Los cuidados sanitarios, cuando excepcionalmente han de ser aplicados por técnicas especiales, pueden perder su carácter de obligatoriedad y convertirse en cuidados extraordinarios o desproporcionados.

En el caso de los pacientes en estado terminal y tratados en una UCI, hay algunos autores que señalan que la futura calidad de vida es la referencia que determina si a un paciente se le deben aplicar tratamientos o incluso esos cuidados mínimos. Si se adopta tal principio, la diferencia entre no cuidar a un 
paciente y la eutanasia directa difícilmente podrá ser mantenida.

Se estima que los cuidados básicos o mínimos para mantener la vida humana son la hidratación y la alimentación. «De toda persona humana se espera moralmente que ayude a otro ser humano en situación de malestar. Un paciente terminal no es una excepción. El médico, la familia, los amigos, las enfermeras, los trabajadores sociales y otros individuos cercanos al paciente moribundo están obligados a proveer ayuda o soporte incluyendo cuidado psicosocial y emocional hasta el final. La alimentación y nutrición son parte y parcela de ese cuidado que no se distingue del acompañar, hablar, cantar, leer o simplemente escuchar al paciente moribundo. Hay veces en las que la específica terapia médico-quirúrgica no está indicada por más tiempo como apropiada o deseable para un paciente terminal. En ninguna ocasión, sin embargo pueden abandonarse las medidas de soporte general acelerándose por lo tanto la muerte del paciente» (13).

En opinión de Martínez Baza, la hidratación por sonda nasogástrica o por vía endovenosa (sin referirnos a la vía oral), son habitualmente beneficiosas para un enfermo terminal. En primer lugar, ayudan a mantener ciertas funciones que en un estado de alteración del equilibrio hidroelectrolítico no se conservarían. Nos referimos, por ejemplo, a la función renal o al estado de alerta mental. En ambos casos es indudable que contribuyen al bienestar del paciente. También contribuyen a aliviar ciertos síntomas que causan malestar al paciente (dificultad para eliminar secreciones bronquiales, sed o complicaciones provocadas por sequedad de mucosas, por ejemplo). En último lugar, es indudable que colaboran y ayudan psicológicamente evitando que el paciente se sienta abandonado (14).

Sin embargo, en el caso del paciente en estado agónico que habitualmente es incapaz, se producen alteraciones fisiológicas que hacen difícil el equilibrio electrolítico, por lo que la hidratación por cualquiera de las vías no aportará beneficios y se convertirá en un cuidado extraordinario, tal vez solamente justificable para que la familia no considere que existe un abandono del moribundo.

En el caso del paciente en estado vegetativo permanente (EVP) por lesión cerebral, la situación es muy distinta, pues no se trata de un paciente terminal en el sentido anteriormente definido, aunque sea incapaz. En este caso, la hidratación por sonda o por vía endovenosa otorgará los beneficios antes señalados y las cargas para el paciente y el personal sanitario serán mínimas, por lo que podemos considerarlo siempre un cuidado básico o mínimo en todos los casos.

\section{La alimentación en el enfermo terminal}

Respecto a la alimentación es preciso matizar algo más. Se puede considerar la alimentación como un cuidado mínimo, pero no necesariamente de cualquier modo. Sería un cuidado ordinario alimentar por sonda nasogástrica y si se espera una supervivencia razonable podría también incluirse la alimentación por gastrostomía. Sin embargo, la alimentación parenteral de hidratos de carbono, lípidos y proteínas, habitualmente se puede considerar como un cuidado extraordinario, pues plantea mayores problemas de aplicación, su costo es elevado, el paciente ha de estar hospitalizado, debe ser controlada por personal médico y no está exenta de riesgo.

En los pacientes en estado agónico, no es obligada la alimentación. En los pacientes en EVP, la alimentación por sonda o gastrostomía se considera cuidado ordinario y, por vía endovenosa, extraordinario. La obligatoriedad o no de estos cuidados, según sean o no ordinarios, recae tanto sobre la familia como sobre el personal sanitario que atiende a esos pacientes.

Los que atienden al paciente han de tener en cuenta algunos principios: 
- que conforme se acerca un paciente a la muerte se vuelve cada vez más desinteresado por la comida y, en menor medida, en la toma de líquidos.

- que los inapetentes son incapaces de disfrutar de una comida abundante.

- que sus necesidades calóricas son ahora mucho menores.

- que no está comprobado que una terapia nutricional agresiva en estos enfermos pueda mejorar su calidad de vida.

Es necesario valorar las causas de los trastornos del apetito y tener en cuenta, en lo posible, las preferencias del paciente, presentación y preparación de las comidas, horarios, dietas especiales, etc. Otra forma importante de ayudarles es dar poco énfasis a su pérdida de peso.

\section{El empleo de medios terapeúticos «desproporcionados»}

¿Hasta qué punto hay que agotar con un enfermo todos los medios terapéuticos? ¿Es ético emplear curas costosas y difíciles aunque den sólo una pequeña esperanza de éxito? ¿Es obligatorio poner todos los medios disponibles en cada caso para mantener a un enfermo en vida el mayor tiempo posible? ¿Se puede o se debe prolongar artificialmente la vida?

A la hora de encontrar solución a estos problemas se afirma que no siempre hay que recurrir a toda clase de remedios posibles. «Hasta ahora los expertos solían responder que no siempre se está obligado al uso de remedios «extraordinarios» para mantener la vida. Actualmente, en cambio, tal respuesta, siempre válida en principio, puede parecer tal vez menos clara, tanto por la imprecisión del término como por los rápidos progresos de la terapia. Por este motivo se prefiere hablar de medios «proporcionados» y «desproporcionados». Para valorarlos habrá que tener en cuenta el tipo de terapia, el grado de dificultad y de riesgo que comporta, los gastos necesarios, y las posibilidades de aplicación con el resultado que se puede esperar de todo ello, teniendo en cuenta las condiciones del enfermo y de sus fuerzas físicas y morales. Con estos elementos habrá datos suficientes para decidir un tratamiento que prolongue la vida o para conformarse con los medios normales que la Medicina puede ofrecer» (16).

Los interrogantes citados se plantean frecuentemente en los medios de difusión, en los planteamientos pro-eutanasia, etc. Aunque no se puede generalizar, en nuestra opinión, el problema afecta más a enfermos que reciben terapias intensivas que a pacientes en situación terminal en general o que están en atención domiciliaria. Entre los profesionales que se dedican a los cuidados paliativos, quizá por la aportación que desde hace años viene haciendo el movimiento «Hospice», parece que estos dilemas no lo son tanto. Aplicando el principio terapéutico o de beneficencia, que lleva a enjuiciar cada situación buscando el bien del enfermo, y el principio de autonomía, que lleva a respetar el derecho del paciente a intervenir en la toma de decisiones sobre el tratamiento a realizar, se puede encontrar una solución ética para cada situación concreta.

Por último, cabe señalar que, desde el punto de vista ético, también es aceptable la postura contraria. A falta de otros remedios y de acuerdo con el paciente, es lícito recurrir a los nuevos medios que ofrece una medicina de vanguardia, aunque todavía se encuentren en fase experimental y no estén libres de todo riesgo; con esa actitud el enfermo podrá, incluso, dar ejemplo de generosidad para el bien de la humanidad.

\section{La certificación de la muerte cerebral}

Es la situación final en el tratamiento al paciente terminal y también incluye una serie de aspectos éticos importantes. Certificar bien y conforme con los criterios establecidos para determinar la muerte de todo el cerebro, es un deber ético para con el paciente. En este sentido, algunas de las nuevas teorías 
neocorticalistas no tienen un debido respeto ético al paciente: suponer que se puede adelantar la certificación de la muerte a que esté dañada solamente la corteza cerebral, supone un enorme riesgo en el caso de pacientes en coma vegetativo persistente que pudieran ser utilizados para donación de órganos o en el caso de niños anencefálicos (17).

Es necesario, en nuestra opinión, certificar de modo absoluto la muerte de todo el cerebro, dentro de ese proceso en que consiste el morir.

\section{Los derechos del enfermo terminal}

Señalaremos solamente algunos de los aspectos más importantes que deben tenerse en cuenta desde la atención de enfermería.

7.1 Intervención del enfermo en la elección del tratamiento. Como ya se ha expuesto, una de las líneas éticas del cuidado al paciente en situación terminal marca la necesidad de no reducir al enfermo a ser simplemente «paciente» (no-agente) en su enfermedad. En este sentido es fundamental su intervención en la toma de decisiones que le atañen. Es lo que se ha dado en llamar «doctrina del consentimiento informado» (18).

El respeto a la autonomía del paciente lleva a realizar algunas consideraciones:

a) Los pacientes tienen autoridad ética y legal para renunciar a algunos o todos los cuidados. Se considera «renuncia no voluntaria» de un tratamiento de sostenimiento a la que se produce cuando un paciente no da un efectivo consentimiento ni un rechazo. A menudo surge porque la capacidad de tomar decisiones es inadecuada $\mathrm{y}$, entonces, un representante tendrá que decidir de acuerdo a los supuestos intereses del paciente.

b) La autodeterminación habitualmente obliga al equipo médico a otorgar los cuidados elegidos por el paciente, excepto cuando estos vayan contra la práctica médica acostumbrada, es decir, cuando supongan intervenciones que a su juicio son contraterapéuticas.

c) Si bien los pacientes no toman las decisiones independientemente de las opiniones de otros, no deben sufrir influjos tales que su libre elección sea impedida. En muchas situaciones de cuidados de salud, los pacientes son en alto grado dependientes de los profesionales de la salud; esta disparidad crea una obligación para el equipo médico de reducir la tendencia de algunos pacientes a actuar bajo una equivocada comprensión de su situación o con un sentimiento de impotencia, de modo que los individuos puedan ejercer efectivamente la autodeterminación.

d) Es inaceptable que un paciente capaz de efectuar elecciones informadas sea excluido del proceso de decisión, pues se lesiona el principio de autodeterminación y se permitiría a terceros influir sobre la duración de su vida o ponerle eventuales cargas. Obviar la elección del paciente puede ser, en muchos casos, más grave que enfrentar al paciente con elecciones difíciles. La única excepción será cuando libremente el paciente delega la capacidad para tomar decisiones en otras personas.

e) Para el real y efectivo ejercicio de la autodeterminación, los profesionales de la salud deben lograr que los pacientes comprendan su actual estado médico, incluida la probable evolución si no se efectúa el tratamiento: que conozcan las intervenciones que podrían serle de ayuda, incluida una prescripción de los procedimientos, la probabilidad y efecto de los riesgos asociados y de los beneficios; por último, que sepan cuál es la opinión del médico.

En definitiva, la cuestión no es simplemente informar al paciente de su enfermedad, sino más bien hacerle participar en la elección de las posibles terapias. En todo caso, la verdad debe ser dada a conocer con prudencia y cuidado, evitando que provoque un indebido trauma psíquico.

7.2 El derecho del enfermo a la verdad. Una de las cuestiones más difíciles en la toma de 
decisiones en el trato con el enfermo en situación terminal es la que se refiere al derecho a conocer la verdad de la enfermedad que padecen. No porque alguien niegue tal derecho, que todos reconocen, sino porque a él se añade, y con frecuencia se contrapone, otro elemento que viene a complicar las cosas: el de suponer que el enfermo no está preparado para recibir una noticia que podría serle contraproducente y, en consecuencia, se le oculta la realidad de su mal. Es decir, se acepta en principio que todo enfermo tiene derecho a estar informado de su dolencia; pero como en la práctica se supone que la verdad puede resultar perjudicial, se opta por no dar información.

La comunicación con el enfermo es una parte fundamental del acto médico. El médico informa al enfermo sobre su padecimiento y es entonces cuando el paciente tiene oportunidad de solicitar información sobre aspectos de su interés. También intervienen los profesionales de enfermería que le atienden y deben tener una buena comunicación con el médico para trasmitir al enfermo la información adecuada. En la comunicación con el enfermo en situación terminal, el médico y la enfermera deben llevar a cabo una actividad altamente personalizada, singular para cada paciente, de acuerdo con su condición y sus propias características. Un aspecto de importancia capital es que el propio paciente actúa como regulador de la cantidad de información que desea recibir. Ahora bien, cuándo y cómo hacerlo; constituyen elementos de una práctica en la que no pueden darse pautas y en la cual no sólo el lenguaje verbal forma parte de la comunicación (19).

Manifestar la verdad supone el compromiso previo de ayudar a asimilarla, de compartir las preocupaciones que surgen, de acompañar en la soledad interior de esos difíciles momentos. Esto implica prudencia, tacto y discreción para detectar el modo, las circunstancias y el momento más adecuado y oportuno. De esto existe ya abundante experiencia en enfermos oncológicos, por ejemplo.
Una cuestión que sí es necesario prever por parte del equipo sanitario que atiende e informa a un enfermo crítico, es decidir quién debe dar la información y que la conozcan todos los miembros del equipo que deben tratar a ese enfermo concreto: médico especialista, enfermera, médico que estará de guardia el fin de semana; así se evitará que el enfermo reciba, en ocasiones, versiones distintas de su estado por parte de diferentes profesionales.

Una situación frecuente y éticamente reprobable, es la omisión de la verdad a un paciente que quiere conocerla. En estas condiciones, mantener el engaño va creando una barrera entre médico y paciente; el primero espacia el contacto con el enfermo al no tener explicaciones lógicas de la creciente sintomatología y el enfermo va perdiendo confianza en la persona que no fue capaz de darle lo que demandaba, sintiéndose así defraudado.

Defender el ideal de una información sincera no supone, sin embargo, convertirla en una obligación absoluta. Lo ideal es la información incluso a aquellos que se niegan a recibirla, pero respetando su voluntad. Se les puede preparar con enorme delicadeza, en el momento oportuno y sin dar una cantidad de información que no podrían asumir. Todo enfermo consciente intuye de alguna manera su gravedad; capta por una serie de síntomas que la evolución de su proceso se hace preocupante.

El aspecto de la esperanza es fundamental. El médico necesita que el paciente la mantenga. Esto no significa que se vayan a ofrecer garantías de recuperación porque sería alentar falsas esperanzas, pero sí lograr mantener una tenue luz que ayude a mantener la comunicación interpersonal. Nunca se puede mentir a un paciente, pero tampoco es absolutamente imprescindible decirle toda la verdad (20).

7.3 El derecho a la asistencia familiar, psicológica y espiritual. Como ya indicamos al hablar de las líneas éticas de la atención al enfermo en situa- 
ción terminal, ésta debe abarcar todos los aspectos vitales del paciente. De este modo, al considerar también los aspectos familiares, psicológicos y espirituales del individuo, se valora a la persona en toda su extensión y se le ayuda a afrontar con profundidad una muerte verdaderamente digna.

Desde luego, la mejor situación para un enfermo terminal es la atención docimiliaria, de modo que pueda seguir esos últimos momentos rodeado de su entorno familiar (21).

Es necesaria también una asistencia psicológica, social y espiritual que permita valorar las necesidades desde el punto de vista personal del propio enfermo terminal y prestarle la ayuda necesaria en cada aspecto. Es un deber ético acercarse al punto de vista del paciente, conocer y respetar sus necesidades, su estado emocional y la forma en que experimenta y afronta su propia situación. En muchas de las páginas de la Dra. C. Saunder, pionera del movimiento «Hospice», y también en las de E. Kübler-Ross son continuas las referencias a la influencia de los valores espirituales en el cuidado terminal y ante la muerte.

La posibilidad de que el enfermo en situación terminal reciba o no una atención espiritual en consonancia con sus propias creencias y deseos, depende, muchas veces de una decisión médica. El descuido, la inadvertencia, el miedo a la reacción del enfermo o de su familia o, simplemente, la proyección sobre el paciente de las propias ideas, pueden condicionar este derecho del paciente que es deber para el médico. La facilitación de este tipo de asistencia, no olvidándola, ni sintiéndose incómodo ante ella, debe estar presente en la mente del médico y de la enfermera.

$Y$ es necesaria también una formación de la enfermera (22) que le ayude a afrontar estos aspectos del sentido del dolor y la muerte, a establecer esa «relación de ayuda» imprescindible para la humanización de los cuidados que van a ayudar al enfermo terminal en esos momentos tan importantes de su vida.

\section{Referencias:}

1 J. van der Aren A. La participación de la enfermera en la parte final de la vida. Consejo Internacional de Enfermería. Enfermería Actualidad 1997;IX:12.

2 Astudillo W, Mendinueta C, Astudillo E. Cómo ayudar al paciente en sus últimos días. Medicina y Persona 1992 ene-mar:33.

3 Vega J, Centeno C, Martínez León M, Martínez Baza P. Bioética de la situación terminal de enfermedad. V Jornada de la S.E. de Medicina Legal y Forense; 1992 Oct 7-9; Córdoba, España.

4 Astudillo, ob.cit.: 33-36.

5 Nieves Martín E. La relación clínica con el paciente difícil. Documentos SEMFYC 7. Sobre Bioética y Medicina de Familia. Palma de Mallorca: SEMFYC; 1996:63-7.

6 Kass LR. Eutanasia y autonomía de la persona: vivir y morir con dignidad. Human Life Review 1989;XVI.

7 Vega J y o tros, ob.cit.: 5.

8 Twycross. Manejo terapéutico del dolor en el cáncer. Trad. de Lab. Sarget; original en Primary Care \& Cancer 1988;V.

9 Espejo Arias MD. Los cuidados paliativos. 1997: 132.

10 Gutiérrez M, De la Fuente M. Problemas éticos de enfermería en Unidades de Cuidados Intensivos. Cuadernos de Bioética 1994;3:182-5

11 Congreso Mundial de Medicina y Cuidados Intensivos. Declaración de Ética en Medicina Crítica y Cuidados Intensivos. Madrid; 1996.

12 Martinón JM, et al. Algunas reflexiones sobre ética en Cuidados Intensivos Pediátricos. Cuadernos de Bioética 1996;26(2):149155.

13 Rossner F. Withdrawing fluids and nutrition: an alternate way. Bulletin of the New York Academy of Medicine 1988 Jun;64(5):363-75.

14 Martínez Baza P, Vega Gutiérrez J, Centeno C. Bioethics of nutrition and hydration in oncological patients en a terminal situation. IX Simposium de la Org. Europea para Prevención del Cáncer; 1991 Oct 17-19, Madrid, España.

15 Astudillo W, Mendinueta C, Tamés J. La alimentación en el enfermo terminal: consejos dietéticos. Rev. Medicina, Universidad de Navarra 1992;IV-VI:31-7.

16 Vega J y o tros. ob.cit.: 7.

17 Colomo J. Muerte cerebral: biología y ética. Eunsa; 1993.

18 President's Commission for the Study of Ethical Problems in Medicine and Biomedical and Behavioral Research. Deciding to Life Sustaining Treatment. Washington: Government Printing; 1982, y Making Health Care Decision. Washington: Government Printing; 1983.

19 Centeno C, Núñez Olarte JM. Revelación del diagnóstico a enfermos oncológicos en situación Terminal. Cuadernos de Bioética 1996;2:156-63.

20 Hennezel M de. La muerte íntima. Barcelona: Plaza y Janés; 1996.

21 Boyero B. Ética de la relación enfermo terminal y entorno familiar. Cuadernos de Bioética 1996;2:168-72.

22 Bermejo JC. Humanizar la salud. Humanización y relación de ayuda en enfermería. San Pablo, Madrid; 1997. 